\title{
Análise paramétrica de um painel com enrijecedor sob flambagem elástica
}

\author{
Parametrical analysis of a stiffened plate under elastic buckling \\ P. B. Rackow ${ }^{1 *}$; M. de V. Real ${ }^{2}$; L. A. Isoldi ${ }^{2}$, R. Colares $^{3}$, D. Thormann ${ }^{3}$ \\ ${ }^{l}$ Programa de Pós-Graduação em Engenharia Oceânica (PPGEO), Universidade Federal do Rio Grande (FURG), \\ 96203-900, Rio Grande-RS, Brasil \\ ${ }^{2}$ Escola de Engenharia (EE), Universidade Federal do Rio Grande (FURG), 96203-900, Rio Grande-RS, Brasil \\ ${ }^{3}$ Programa de Pós-Graduação em Modelagem Computacional (PPGMC), Universidade Federal do Rio Grande \\ (FURG), 96203-900, Rio Grande-RS, Brasil \\ *patricia.rackow@yahoo.com.br \\ (Recebido em 18 de setembro de 2014; aceito em 16 de outubro de 2014)
}

\begin{abstract}
As estruturas navais e offshore são constituídas, basicamente, de painéis enrijecidos. Quando submetidos a carregamentos compressivos, surge a preocupação em relação ao comportamento desses em relação à flambagem. $\mathrm{O}$ fenômeno da flambagem ocorre em peças esbeltas que, quando submetidas à compressão axial, após atingida a carga crítica, sofrem uma deflexão transversal. Peças sob flambagem podem perder sua estabilidade sem que o material tenha atingido sua tensão de escoamento. $\mathrm{O}$ objetivo do presente trabalho é realizar, através de modelagem computacional, a análise linear de um painel quando submetido à flambagem elástica. Esse painel é constituído por uma chapa plana e um reforço no formato de barra. Através de um modelo computacional desenvolvido no software ANSYS®, o qual se baseia no Método dos Elementos Finitos, foi feita a variação dos parâmetros geométricos desse painel, sendo possível avaliar a influência do aumento da espessura da placa, espessura do enrijecedor e altura do enrijecedor no valor da tensão crítica de flambagem do painel. Os resultados mostram a grande influência da presença dos enrijecedores na determinação da carga crítica.
\end{abstract}

Palavras-chave: painel enrijecido, flambagem, Método dos Elementos Finitos

Naval and offshore structures are formed, basically, by stiffened plates. When under compressive loads, there is the concern about the behavior of these plates in relation to buckling. Buckling phenomenon happens in slender members that, subjected to to axial compression, when a critical load is reached, suddenly suffer a lateral deflection. Members under buckling may lose its stability before the material has reached its yield stress. The objective of this paper is to carry out, by computational modeling, a linear analysis of a stiffened plate under elastic buckling. This panel is formed by a plate and one bar-stiffener. Through a computational model developed in ANSYS® software, which is based on the Finite Element Method, a parametric variation on the plate geometry was made. Through this parametric study it was possible to evaluate the influence of increasing plate thickness, stiffener thickness and stiffener height on critical buckling load of the panel. Results show the huge influence of stiffeners on critical load.

Keywords: stiffened plate, buckling, Finite Element Method

\section{INTRODUÇÃO}

Estruturas navais e offshore exigem membros estruturais relativamente esbeltos e capazes de resistir a grandes carregamentos. Sendo assim, a relação resistência/peso torna-se muito importante.

Os painéis são componentes estruturais constituídos por paredes finas com uma elevada relação entre resistência e peso, apresentando vantagens em seu uso devido ao baixo custo de fabricação e à facilidade de construção, dados a partir de processos de corte e soldagem.

Devido à esbeltez destas placas, faz-se necessária a utilização de reforços, aumentando sua capacidade de carga.

Os painéis reforçados por enrijecedores, Figura 1, são constituídos por chapas e reforços longitudinais, que geralmente são colocados com espaçamento constante entre si e perpendiculares ao bordo do painel, acompanhando a direção dominante da carga. 


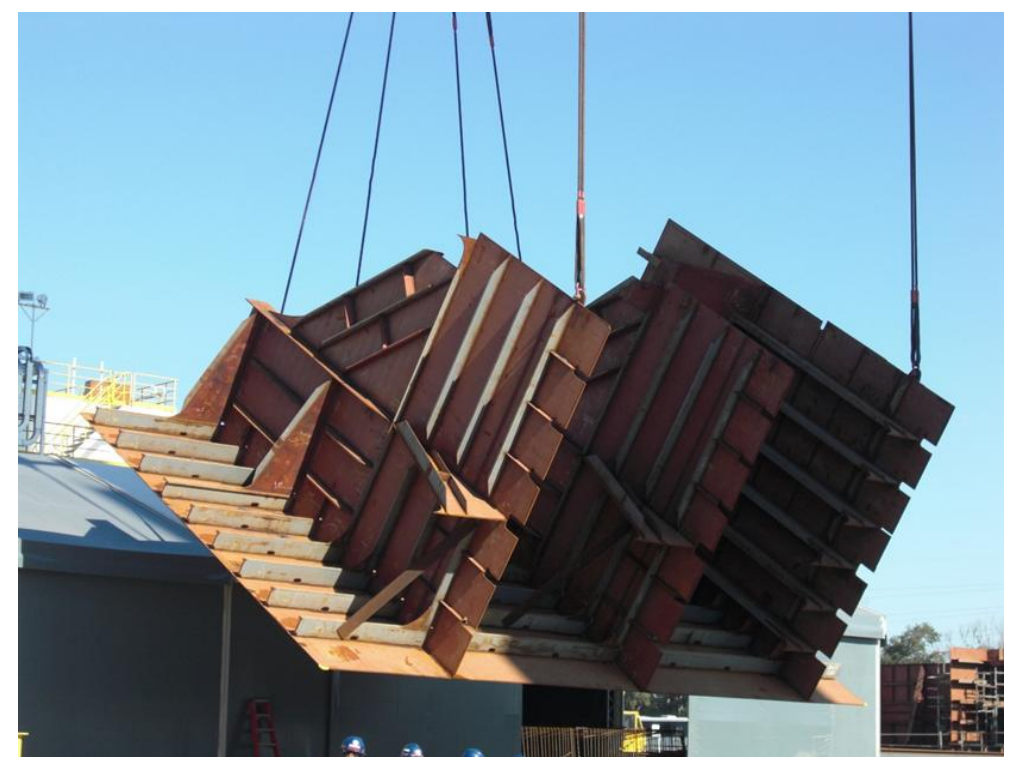

Figura 1: Painéis enrijecidos utilizados em plataformas de extração de petróleo.

A Figura 2 mostra os tipos de reforços mais utilizados para enrijecimento de placas planas em estruturas de navios.

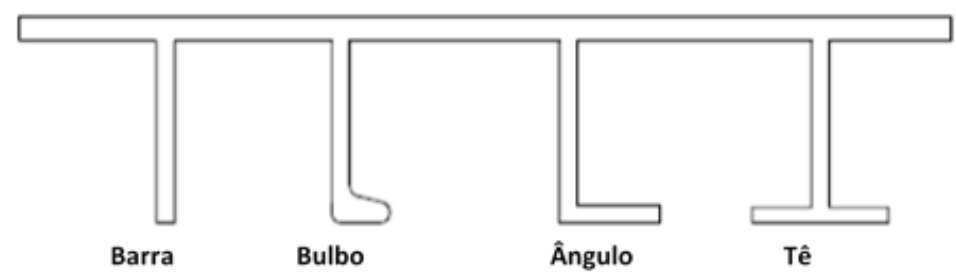

Figura 2: Tipos de reforços mais comuns em placas planas.

Dependendo das solicitações às quais o navio está submetido, os painéis que o compõem podem sofrer tração ou compressão. Tensões de compressão fazem com que estes painéis esbeltos sofram os efeitos da flambagem.

O fenômeno da flambagem ocorre em peças esbeltas que, quando submetidas à flexão transversal devido à compressão axial, sofrem uma deflexão. Por se tratarem de elementos bidimensionais, a flambagem em placas envolve flexão em dois planos. Peças sob flambagem podem perder sua estabilidade sem que o material tenha atingido sua tensão de escoamento, sendo, por isso, considerada uma instabilidade elástica.

Chajes [1] ressalta as diferenças entre a flambagem de colunas e de placas. Colunas sob flambagem não são mais capazes de resistir à carga axial e, portanto, a carga crítica é igual à carga de ruptura do membro. Para placas, esta teoria não é aplicável. Estes elementos, mesmo após terem atingido sua carga crítica, podem continuar resistindo a um aumento de carga axial sem apresentar falhas, até que esta seja consideravelmente maior que sua carga crítica. Desta forma, a carga crítica não é igual à carga de ruptura.

Ele realizou a análise do comportamento pós-flambagem de placas comprimidas axialmente e chegou às seguintes conclusões:

1. Placas possuem resistência pós-flambagem, a qual permite o aumento de carga após atingir o valor crítico.

2. As tensões de tração transversais que surgem após o início da deformação das placas são o principal fator responsável pela resistência pós-flambagem.

3. As áreas próximas às bordas longitudinais das placas resistem à maior parte do aumento de carga que ocorre na fase pós-flambagem.

Painéis com enrijecedores podem falhar de acordo com uma das formas ilustradas na Figura 3. 


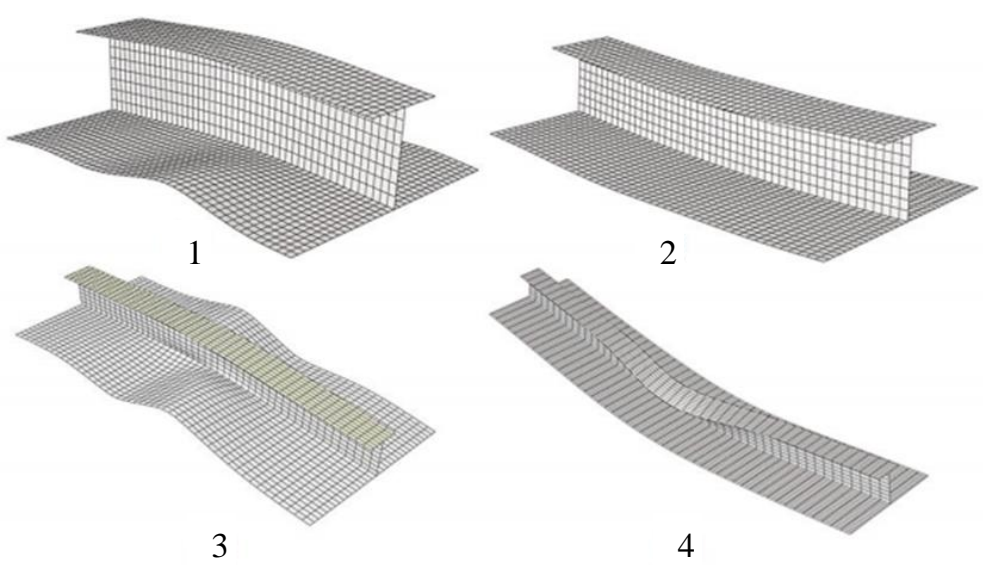

Figura 3: Tipos de flambagem em painéis com enrijecedores.

1. Flambagem global induzida pela placa: falha simultânea do reforço e da placa, provocada por uma flambagem inicial da placa. Após a falha, o reforço encontra-se no lado convexo da chapa.

2. Flambagem global induzida pelo reforço: falha simultânea do reforço e da placa, provocada por uma flambagem inicial no reforço. Após a falha, o reforço encontra-se no lado côncavo da chapa.

3. Flambagem da placa: flambagem local da placa, sem afetar os reforços, que permanecem retos, ocasionando uma redistribuição de carga entre os mesmos. Ocorre quando a chapa tem uma tensão crítica inferior à do reforço e/ou o reforço é muito robusto.

4. Flambagem torcional dos reforços (tripping): rotação do reforço no local onde o mesmo é unido à chapa. É considerado o mais perigoso, uma vez que leva a estrutura à uma queda brusca e repentina da capacidade de carga, devido à falta de rigidez torcional do reforço.

Diversos pesquisadores desenvolveram trabalhos referentes a painéis com enrijecedores. Taysi [2] propôs determinar a espessura e a localização dos enrijecedores para otimização da carga crítica em placas enrijecidas. Para isto, foram analisados dois perfis de placas comuns na indústria aeroespacial. $\mathrm{O}$ autor concluiu que o aumento da carga crítica é pequeno quando é feita apenas a otimização de tamanho. No entanto, na otimização do formato esses resultados são melhores. A melhoria na carga crítica mais significante é dada quando é feita a otimização de tamanho e formato juntamente.

Em relação à localização dos reforços, o resultado ficou em função das condições de contorno. O aumento na quantidade de enrijecedores leva a um aumento considerável de carga crítica.

Tanto o estudo paramétrico quanto o estudo de otimização mostraram que a carga crítica varia bastante de acordo com a localização dos enrijecedores.

Chujutalli [3] analisou a flambagem torcional dos reforços de painéis, chamada de tripping, que é o mais perigoso modo de falha, já que acontece repentinamente. Para tal, realizou um estudo paramétrico para diferentes relações geométricas dos enrijecedores, variando-se individualmente as espessuras da chapa, da alma e do flange dos reforços.

Observou-se que a diminuição da espessura da chapa reduz consideravelmente a resistência máxima da mesma, apresentando pouca influência nos reforços.

A variação da espessura da alma do reforço mostrou que a menor espessura leva à falha local na alma do mesmo, reduzindo drasticamente sua resistência e, posteriormente, gerando tripping. Porém, para as outras espessuras, o primeiro modo de falha apresentado é a flambagem da chapa, seguido do tripping.

$\mathrm{Na}$ análise da variação da espessura do flange do reforço, notou-se pouca influência na resistência máxima, mas o efeito é considerável no seu comportamento pós-flambagem.

O objetivo principal deste trabalho é avaliar, através de modelagem computacional, o comportamento estrutural de um painel reforçado sob flambagem elástica, variando seus parâmetros geométricos e observando a influência no valor da tensão de flambagem. 
A variação paramétrica proposta envolve os dados da geometria da chapa e do reforço, que são: espessura da placa, espessura do enrijecedor e altura do enrijecedor.

Esta modelagem foi feita no software ANSYS ${ }^{\circledR}$, o qual permite a análise de painéis pelo Método dos Elementos Finitos (MEF).

\section{MATERIAL E MÉTODOS}

O painel analisado é formado por uma chapa plana retangular de comprimento $a$, largura $b \mathrm{e}$ espessura $t_{p}$ com um enrijecedor longitudinal de altura $h_{w}$ e espessura $t_{w}$ posicionado em ${ }^{b} / 2$, conforme a Figura 4.

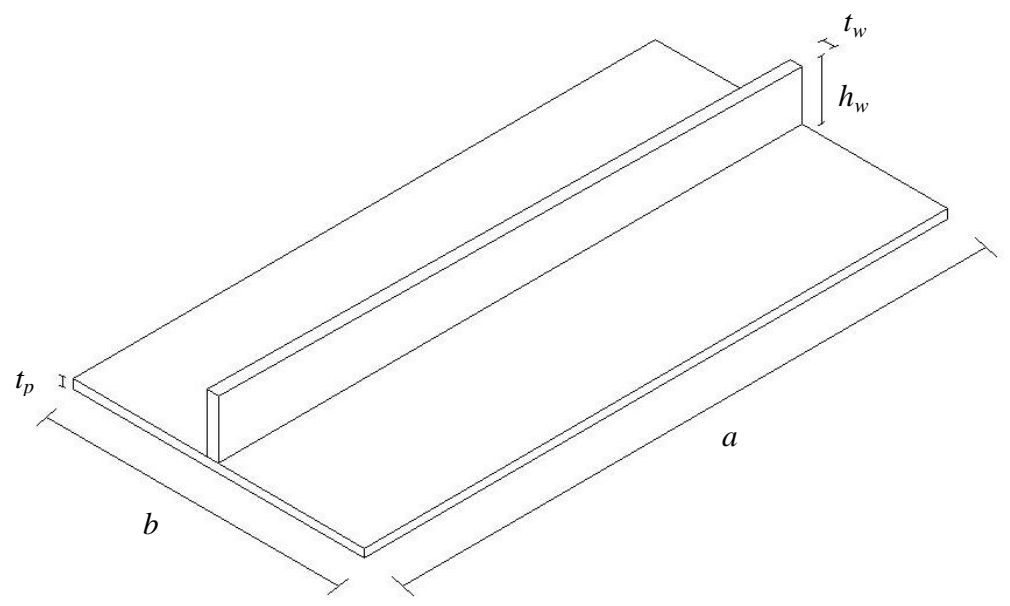

Figura 4: Geometria do painel com um enrijecedor.

Esse painel reforçado, simplesmente apoiado, foi modelado no software ANSYS ${ }^{\circledR}$, que se baseia no Método de Elementos Finitos (MEF) e é amplamente usado para solucionar problemas de engenharia.

O MEF é utilizado para obter soluções aproximadas para diversos problemas em análises de engenharia com uma considerável precisão. O método consiste em dividir uma estrutura contínua em diversos elementos finitos. Esses elementos estão interconectados entre si, através de nós localizados em seus limites, e o comportamento de cada um deles é determinado de forma que a malha formada pelo conjunto se comporte de forma semelhante ao contínuo original.

Para determinar os deslocamentos nodais, existe uma série de equações algébricas de equilíbrio, formando um sistema de equações equivalente à malha utilizada. A solução pode ser obtida aplicando-se condições externas.

$\mathrm{Na}$ modelagem computacional é adotada a análise elástica de autovalores. Segundo Madenci e Guven [4], essa análise envolve a solução de um conjunto de equações algébricas homogêneas cujo menor autovalor corresponde à carga crítica de flambagem e o autovetor associado representa o modo primário de flambagem.

A matriz de rigidez total $[K]$ é obtida através da soma da matriz de rigidez convencional para pequenas deformações $\left[K_{E}\right]$ com a matriz de rigidez geométrica $\left[K_{G}\right]$, que não depende apenas da geometria, mas também do esforço interno existente no início do carregamento $\left\{P_{0}\right\}$. Desta forma, a matriz de rigidez total de uma placa para um nível de carga $\left\{P_{0}\right\}$ é dada por:

$$
[K]=\left[K_{E}\right]+\left[K_{G}\right]
$$

onde: $[K]$ é a matriz de rigidez global; $\left[K_{E}\right]$ é a matriz de rigidez convencional para pequenas deformações e $\left[K_{G}\right]$ é a matriz de rigidez geométrica.

Quando a carga atinge o nível de $\left\{P_{0}\right\}=\lambda\left\{P_{0}\right\}$, onde $\lambda$ é um escalar, a matriz de rigidez pode ser definida como: 


$$
[K]=\left[K_{E}\right]+\lambda\left[K_{G}\right]
$$

Assim, as equações de equilíbrio governantes para o comportamento da placa podem ser escritas como:

$$
\left[\left[K_{E}\right]+\lambda\left[K_{G}\right]\right]\{U\}=\lambda\left\{P_{0}\right\}
$$

onde: $\{U\}$ é o vetor de deslocamentos total, que pode ser determinado por:

$$
\{U\}=\left[\left[K_{E}\right]+\lambda\left[K_{G}\right]\right]^{-1} \lambda\left\{P_{0}\right\}
$$

Sob flambagem, a placa apresenta um grande crescimento nos seus deslocamentos sem aumento de carga. Da definição matemática de matriz inversa como sendo a matriz adjunta dividida pelo determinante dos coeficientes, é possível notar que os deslocamentos $\{U\}$ tendem ao infinito quando:

$$
\operatorname{det}\left[\left[K_{E}\right]+\lambda\left[K_{G}\right]\right]=0
$$

A Equação (5) representa um problema de autovalores, cuja solução gera o menor autovalor, que corresponde à carga crítica em que ocorre flambagem:

$$
\left\{P_{c r}\right\}=\lambda_{1}\left\{P_{0}\right\}
$$

onde: $\left\{P_{c r}\right\}$ é a carga crítica e $\lambda_{1}$ corresponde ao menor autovalor gerado.

Além disso, o vetor de deslocamento escalar associado $\{U\}$ define o formato do membro na flambagem. No ANSYS ${ }^{\circledast}$, o problema de autovalor é resolvido usando o método numérico Lanczos.

O software possui uma base de dados com vários tipos de elementos indicados para diferentes tipos de análises. O elemento escolhido para as análises desse trabalho é o SHELL93, indicado para modelar superficies do tipo casca e tem seis graus de liberdade por nó, que são: translações nas direções $x, y$ e $z$, e rotações em torno dos eixos $x, y$ e $z$.

Para a definição do refinamento de malha a ser adotado, foi feito um teste de convergência, que identifica um tamanho de elemento que seja capaz de gerar um resultado preciso exigindo o menor esforço computacioal possível. O teste consiste em verificar quando duas simulações sucessivas apresentem resultados muito próximos, adotando a máxima diferença relativa da tensão de flambagem permitida como $0,2 \%$, conforme a Equação (7).

$$
\left|\frac{\sigma_{c r}^{n}-\sigma_{c r}^{n-1}}{\sigma_{c r}^{n}}\right| \times 100 \leq 0,20 \% \quad n=1,2, \ldots N
$$

onde: $\sigma_{c r}$ é a tensão crítica.

Considerou-se uma placa de comprimento $a=1000 \mathrm{~mm}$, largura $b=500 \mathrm{~mm}$, espessura $t_{p}=$ $9 \mathrm{~mm}$ com um enrijecedor de altura $h_{w}=50 \mathrm{~mm}$ e espessura $t_{w}=9 \mathrm{~mm}$. O módulo de elasticidade (módulo de Young) e o coeficiente de Poisson do material são, respectivamente, $E$ $=210.000 \mathrm{~N} / \mathrm{mm}^{2}$ e $v=0,30$.

Os resultados para o teste de convergência da malha, bem como os sucessivos refinamentos e diferenças relativas são apresentados na Tabela 1. 
Tabela 1: Teste de convergência de malhas.

\begin{tabular}{|c|c|c|c|c|}
\hline Simulação & Tamanho do & to $(\mathbf{m m})$ & Tensão crítica $\left(\mathrm{N} / \mathrm{mm}^{2}\right)$ & Diferença relativa $(\%)$ \\
\hline \multirow{3}{*}{1} & direção $a$ & 200 & \multirow{3}{*}{638,01} & \multirow{3}{*}{0,38} \\
\hline & direção $b$ & 100 & & \\
\hline & direção $h w$ & 20 & & \\
\hline \multirow{3}{*}{2} & direção $a$ & 100 & \multirow{3}{*}{640,42} & \multirow{3}{*}{0,05} \\
\hline & direção $b$ & 50 & & \\
\hline & direção $h w$ & 10 & & \\
\hline \multirow{3}{*}{3} & direção $a$ & 150 & \multirow{3}{*}{640,77} & \multirow{3}{*}{-} \\
\hline & direção $b$ & 62,5 & & \\
\hline & direção $h w$ & 10 & & \\
\hline
\end{tabular}

Observa-se que os tamanhos de elementos da terceira simulação satisfazem à condição imposta pela Equação (7). Sendo assim, esta foi a malha adotada para as modelagens computacionais apresentadas neste trabalho.

A verificação desta modelagem foi feita considerando as mesmas propriedades geométricas do painel analisado no teste de convergência. A tensão crítica de uma placa retangular simplesmente apoiada com um reforço longitudinal possui uma solução analítica proposta por Timoshenko [5]:

$$
\sigma_{c r}=k \frac{\pi^{2} D}{b^{2} t_{p}}
$$

onde: $\sigma_{c r}$ é a tensão crítica, $k$ é um fator que depende das proporções da placa e do reforço, $\pi$ é a constant numérica igual a 3,14159265 e $D$ é uma variável que depende das propriedades do material e da espessura da placa, conforme a Equação (9).

$$
D=\frac{t_{p} E}{12\left(1-v^{2}\right)}
$$

Para determinar o fator $k$, faz-se necessário calcular as seguintes variáveis:

$$
\begin{aligned}
& \beta=\frac{a}{b} \\
& \delta_{i}=\frac{t_{w} h_{w}}{b t_{p}} \\
& \gamma=\frac{E I}{b D}
\end{aligned}
$$

onde: I é o momento de inércia do enrijecedor.

$$
\begin{aligned}
& c=\left(1+\beta^{2}\right)^{2}+2 \gamma \\
& d=\left(1+9 \beta^{2}\right)^{2}+2 \gamma
\end{aligned}
$$

O valor de $k$ pode ser calculado através da seguinte equação:

$$
\left(k \beta^{2}\right)^{2}(1+4 \delta)-k \beta^{2}[(1+2 \delta)(c+d)-8 \gamma \delta]+c d-4 \gamma^{2}=0
$$

Para o painel com a geometria proposta, a tensão crítica de flambagem determinada pela Equação (8) é $606,12 \mathrm{~N} / \mathrm{mm}^{2}$. Através da modelagem computacional, tem-se a tensão crítica como sendo $640,77 \mathrm{~N} / \mathrm{mm}^{2}$. 
Observa-se uma pequena e aceitável diferença de 5,41\% entre a solução analítica e o modelo computacional proposto, já que a solução analítica trata o problema de forma aproximada.

\section{RESULTADOS E DISCUSSÃO}

As análises foram feitas variando os parâmetros em torno de um modelo tomado como base (placa de comprimento $a=1000 \mathrm{~mm}$, largura $b=500 \mathrm{~mm}$, espessura $t_{p}=9 \mathrm{~mm}$ com um enrijecedor de altura $h_{w}=50 \mathrm{~mm}$ e espessura $t_{w}=9 \mathrm{~mm}$ ). O módulo de elasticidade (módulo de Young) e o coeficiente de Poisson do material de todas as modelagens são, respectivamente, $E=$ $210.000 \mathrm{~N} / \mathrm{mm}^{2}$ e $v=0,30$.

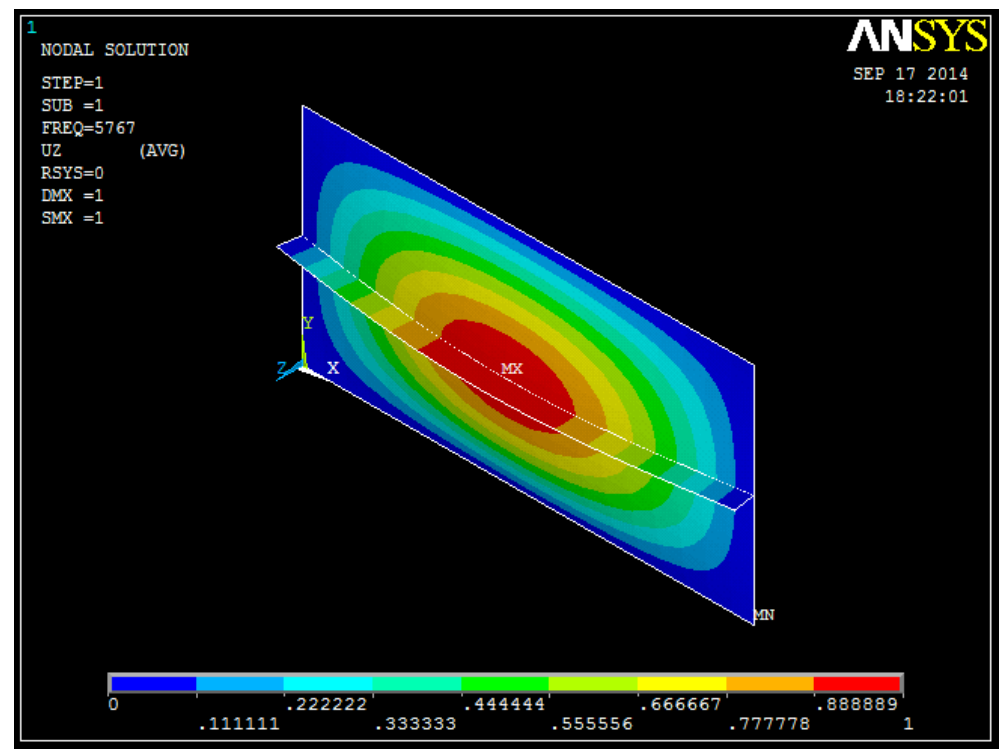

Figura 5: Modo de flambagem elástica do painel base.

Os resultados gerados no ANSYS $^{\circledR}$ para todas as simulações representam uma carga distribuída ao longo da borda da placa. Para encontrar a tensão crítica de flambagem, faz-se necessário dividir esse valor pela espessura $t_{p}$ da placa.

\subsection{PLACA SEM ENRIJECEDOR}

Afim de verificar a eficiência do uso de enrijecedores, foi analisada uma placa plana de comprimento $a=1000 \mathrm{~mm}$, largura $b=500 \mathrm{~mm}$, espessura $t_{p}=9 \mathrm{~mm}$ sem nenhum reforço.

A tensão crítica encontrada é igual a $242,61 \mathrm{~N} / \mathrm{mm}^{2}$, valor expressivamente menor que o obtido para a mesma placa com a presença um enrijecedor.

\subsection{VARIAÇÃO DA ESPESSURA DA PLACA $t_{p}$}

Variando a espessura da placa $t_{p}$, foram encontrados os resultados expostos na Tabela 2.

Tabela 2: Variação da espessura da placa.

\begin{tabular}{cc}
$\boldsymbol{t}_{p}(\mathbf{m m})$ & $\boldsymbol{\sigma}_{c r}\left(\mathbf{N} / \mathbf{m m}^{2}\right)$ \\
\hline 7 & 564,03 \\
\hline 9 & 640,77 \\
\hline 11 & 773,55 \\
\hline 13 & 955,08 \\
\hline 15 & 1180,93 \\
\hline
\end{tabular}


Observa-se um significativo aumento no valor da tensão crítica com pequenos incrementos na espessura da placa. Para todas as espessuras de placa o modo de falha ocorrido foi flambagem global induzida pela placa.

\subsection{VARIAÇÃO DA ESPESSURA DO ENRIJECEDOR $t_{w}$}

Variando a espessura do enrijecedor $t_{w}$, foram encontrados os resultados apresentados na Tabela 3.

Tabela 3: Variação da espessura do enrijecedor.

\begin{tabular}{cc}
$\boldsymbol{t}_{\boldsymbol{w}}(\mathbf{m m})$ & $\boldsymbol{\sigma}_{\boldsymbol{c r}}\left(\mathbf{N} / \mathbf{m m}^{2}\right)$ \\
\hline 7 & 588,77 \\
\hline 9 & 640,77 \\
\hline 11 & 690,04 \\
\hline 13 & 736,87 \\
\hline 15 & 781,44 \\
\hline
\end{tabular}

Pode-se observar que o aumento da espessura do reforço leva a um aumento da tensão crítica, que apresenta maior intensidade para as menores espessuras e diminui de intensidade nas maiores espessuras.

\subsection{VARIAÇÃO DA ALTURA DO ENRIJECEDOR $\boldsymbol{h}_{w}$}

Variando a altura $h_{w}$ do reforço, foram encontrados os seguintes resultados da Tabela 4.

Tabela 4: Variação da altura do enrijecedor.
\begin{tabular}{cc}
\hline $\boldsymbol{h}_{\boldsymbol{w}}(\mathbf{m m})$ & $\boldsymbol{\sigma}_{\boldsymbol{c}}\left(\mathbf{N} / \mathbf{m m}^{\mathbf{2}}\right)$ \\
\hline 40 & 510,28 \\
\hline 50 & 640,77 \\
\hline 60 & 825,28 \\
\hline 70 & 1063,37 \\
\hline 80 & 1069,88 \\
\hline
\end{tabular}

Nota-se que a tensão crítica sofre elevação com o aumento da altura do enrijecedor. Para as alturas de 40, 50 e $60 \mathrm{~mm}$ o modo de falha segue do tipo flambagem global induzida pela placa. Para as alturas de 70 e $80 \mathrm{~mm}$ ocorre uma alteração nesse modo, sendo ocasionado o tripping, ilustrado na Figura 6. 


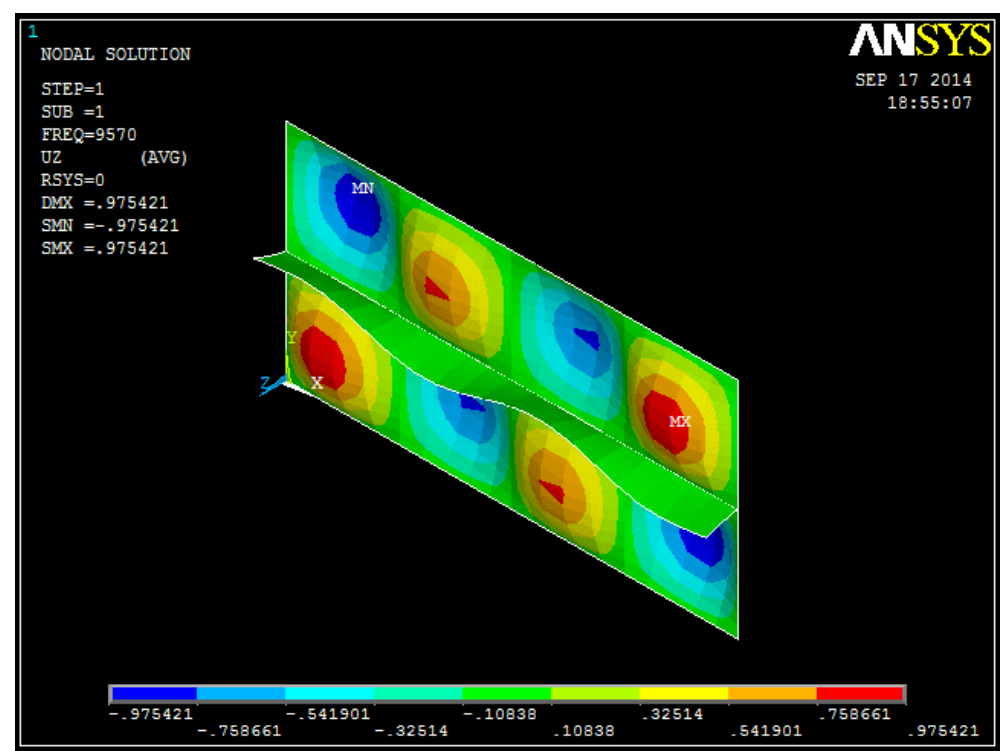

Figura 6: Modo de flambagem elástica para $h_{w}=70 \mathrm{~mm}$

\section{CONCLUSÃO}

A presença de enrijecedores aumentou a tensão crítica de flambagem da placa em $62,14 \%$, confirmando a importância do uso de reforços em placas planas e sua eficiência para aumentar a resistência dos painéis.

A variação na espessura da placa modificou de forma expressiva a tensão crítica. A elevação da espessura do enrijecedor levou a um aumento menos significante na tensão crítica. Esse aumento diminuiu ao passo que a placa atingiu uma espessura suficientemente grande. A tensão crítica aumentou quase que proporcionalmente com o aumento da altura do enrijecedor e apresento influência no tipo de flambagem.

As análises feitas no presente trabalho ratificam a importância de estudar o quanto a variação nos parâmetros geométricos pode influenciar na resistência à flambagem dos painéis utilizados na indústria naval e offshore.

\section{AGRADECIMENTOS}

À CAPES, pela concessão de bolsa de Mestrado no Programa de Pós-Graduação em Engenharia Oceânica, e à FURG e ao CNPq, por darem condições para o desenvolvimento desta pesquisa.

\section{REFERÊNCIAS BIBLIOGRÁFICAS}

1. Chajes A. Principles of structural stability theory. New Jersey: Prentice-Hall; 1974. 336 p.

2. Taysi N. Determination of thickness and stiffener locations for optimization of critical buckling load of stiffened plates. Sci Res Essays. 2010 May;5(9):897-910.

3. Chujutalli JAH. Flambagem torcional de enrijecedores em painéis de navios tanques submetidos à compressão axial. Rio de Janeiro: UFRJ/COPPE; 2010. 161 p.

4. Madenci E, Guven I. The finite element method and applications in engineering using ANSYS®. New York: Springer; 2006. 686 p.

5. Timoshenko SP, Gere JM. Theory of elastic stability. New York: McGraw-Hill; 1963. 541 p. 